\title{
ELECTROCHEMICAL AND IMMUNOELECTRON MICROSCOPY EVIDENCE OF LIPID-PROTEIN INTERACTION IN LANGMUIR-BLODGETT FILMS OF THE HUMAN LUNG SURFACTANT
}

\section{ERNA LADANYI}

Department of Occupational Health, University of Göttingen, Göttingen (F.R.G.)

I. R. MILLER

Department of Membrane Research, The Weizmann Institute of Science, Rehovot (Israel) D. MÖBIUS

Max-Planck Institut für Biophysikalische Chemie, Göttingen (F.R.G.)

RONIT POPOVITS-BIRO

Department of Structure Chemistry, The Weizmann Institute of Science, Rehovot (Israel)

Y. MARIKOVSKY

Department of Membrane Research, The Weizmann Institute of Science, Rehovot (Israel)

P. VON WICHERT AND B. MÜLLER

The Medical Polyclinic, Philipps University, Marburg (F.R.G.)

K. STALDER

Department of Occupational Health, University of Göttingen, Göttingen (F.R.G.)

(Received April 25, 1989; accepted May 2, 1989)

The extracellular lung surfactant surface film (ELSSF) which lines the mammalian lung alveoli at the alveolar air-aqueous cell surface interface is vital in both the breathing and the pulmonary defence processes. The molecular composition of, the structure of and the interaction in the ELSSF was studied, after the ELSSF of human lung lavages could be separated from the subphase and reassembled from its components by using the multicompartment Fromherz-type Langmuir-Blodgett trough. Transmission electron microscopy images of immunogold-labelled and negatively stained isolated film specimens were seen in a continuous layer of mostly phospholipid head groups surfactant-specific protein SpA molecules. Electrical double-layer capacitance and oxygen reduction potential measurements carried out by transferring the surface film from the air-water to a mercury-saline interface of a hanging mercury drop electrode revealed a strong lipid-protein SpA interaction. SpA molecules were partly squeezed out from the film by compression; a proteinless lipid film proved to be a condensed multilayer. Contact with SpA transformed the multilayer into a loose monomolecular film. It is suggested that $\mathrm{SpA}$ molecules play a lipid-transporting role, removing lipids in excess from the air-water interface into the aqueous subphase and vice versa. Lipidprotein interaction can be of importance in vivo. An explanation of how the surfactant film works during the two phases of breathing is proposed. 


\section{INTRODUCTION}

The totality of the mammalian lung alveoli can be regarded as a huge Langmuir trough. The adult human alveolar walls with a total surface area varying between $80 \mathrm{~m}^{2}$ at expiration and $120 \mathrm{~m}^{2}$ at inspiration are covered with a thin aqueous subphase less than $1 \mu \mathrm{m}$ thick. The surface-active components of the subphase-a great variety of phospholipids, neutral lipids ${ }^{1}$ and specific proteins ${ }^{2}$-adsorb after rearrangement at the alveolar air-water interface by building the so-called extracellular lung surfactant surface film (ELSSF). Thus the ELSSF is a biological Langmuir-Blodgett (LB) film. Because it controls the surface tension at the alveolar interface ${ }^{3}$ and a part of it is transferred to the surface of inhaled particles ${ }^{4}$, the ELSSF plays a vital role in both the breathing and the pulmonary defence processes ${ }^{5}$.

Little is known about the actual composition of, the molecular structure of and the molecular interaction in the ELSSF. There are no methods available for in vivo studies. When extracted from the alveoli, the ELSSF is disorganized and its components are mixed with those of the precursors containing the subphase. Thus the data obtained from the extracts cannot be reliable for the ELSSF itself.

By application of the multicompartment Fromherz-type trough to surfactant studies it became possible to isolate the ELSSF from the subphase ${ }^{6}$ in vitro-a prerequisite of any ELSSF study--and to reassemble it from its components ${ }^{7}$. As a result, we visualized the ELSSF of reassembled and crude lung lavages not in crosssection, but en face, i.e. viewed from above and showing transmission electron micrographs of its structure at a molecular level. The present work is part of our project to make a molecular map of the ELSSF and to describe the interactions between its components.

\section{EXPERIMENTAL DETAILS}

The biological materials used in this study consisted of crude human bronchoalveolar lavages obtained from patients, a chloroform-methanol extract of amniotic fluid, purified major surfactant-specific protein $\operatorname{SpA}(M=32-36 \mathrm{kD})$ and polyclonal antihuman SpA antibody from rabbit.

The isolation of the ELSSF from the hypophase was carried out as described earlier $^{6}$. The lavage was deposited in one of the compartments of the Fromherz-type trough (Mayer-Feintechnik, Göttingen, F.R.G.). After achieving adsorption equilibrium the surface film was transported over three compartments filled with aqueous $0.16 \mathrm{M} \mathrm{NaCl}$. The ELSSF over the third compartment was considered to be isolated from the subphase and washed from other non-film-building components.

For reassembly experiments the extracted lipids were spread over a salinecontaining compartment and subsequently shifted over a protein-solutioncontaining compartment. After obtaining surface pressure equilibrium the obtained film was washed by passing it over several saline-filled compartments. The film was washed also after compression experiments.

For the electrochemical measurements the ELSSF was transferred from the air-water interface onto the mercury-saline interface of a hanging mercury drop 
electrode $(\mathrm{HMDE})^{8}$ with and without other previous separation in the trough. Measurements were also carried out at the HMDE-saline interface in the bulk.

Lipid-protein interaction was monitored with a PAR model 170 polarograph (Princeton, NJ, U.S.A.) by electrical double-layer capacitance measurements recording the adsorption a.c., by oxygen half-peak reduction potential measurements recording the triangle d.c. at a HMDE, with a Wilhelmy balance by following the surface pressure changes and by TEM identification (JEM-100 B electron microscope, JEOL, Tokyo, Japan) of SpA in the film specimens lifted on Formvarand Polylysin-coated grids, immuno-gold labelled with $5 \mathrm{~nm}$ gold protein A (Janssen, Belgium) and subsequently negatively stained.

\section{RESULTS AND DISCUSSION}

As expected, the ELSSF is basically a phospholipid film. The electron micrographs of the negatively stained specimens revealed a precursor-free continuous layer in which round electron-translucent individual points of at least three different sizes (aligned as a string of pearls) could be distinguished. The majority of the electron-translucent points occupied an area similar to that found with a dipalmitoylphosphatidylcholine film at the same surface pressure, and isolated and stained in the same way. This result was in agreement with the lavage data, since $90 \%$ of the lavage consists of lipids and $70 \%$ of these are phospholipids.

The immuno-gold-labelled and negatively stained specimens of both the isolated and the non-isolated ELSSFs of the lavage showed randomly distributed dark points among the negatively stained lipid molecules (Fig. 1). The electronopaque dark points are the gold particles indicating SpA. Thus the major surfactantspecific protein SpA found in the whole lavage was either attached or a constituent

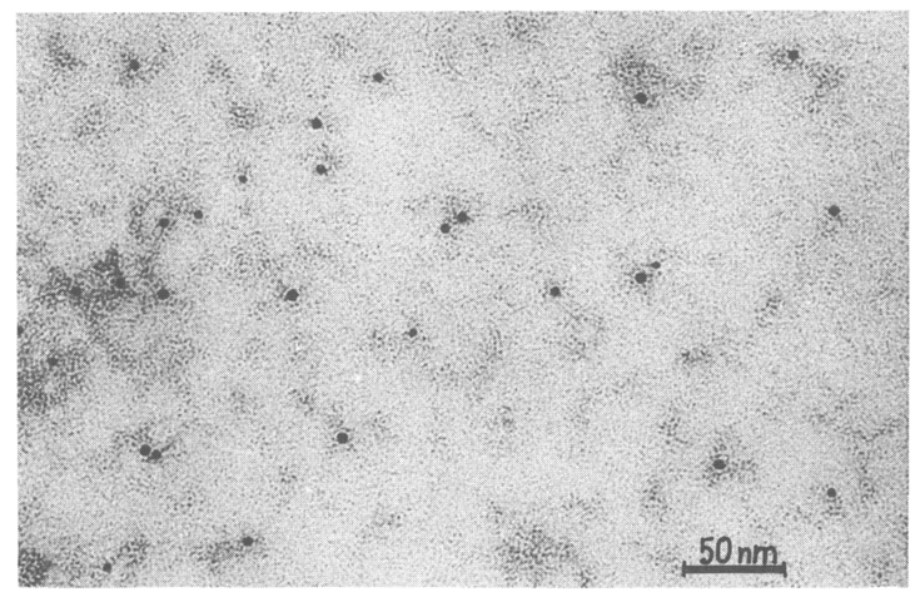

Fig. 1. Transmission electron micrograph of the immuno-gold-labelled and uranylacetate-stained reassembled ELSSF showing the phospholipid head groups (white points) and surfactant-specific SpA molecules (dark points). The penetrating proteins increased the surface pressure from 25.7 to $35.5 \mathrm{~m} \mathrm{~N} \mathrm{~cm}^{-1}$. 
of the surface layer at the air-water interface. However, we also found it in the subphase-isolated ELSSF. We have to keep in mind that SpA is water soluble and that during the process of isolation from the subphase the ELSSF was transported over several aqueous compartments without any essential loss in the film surface pressure. Therefore, we can conclude that not only was $\mathrm{SpA}$ attached to the lipid layer but also it was a constituent of it and that a strong hydrophobic interaction between ELSSF lipids and SpA molecules allowed them to be cotransported together with the lipids.

On the contrary, compression of the film resulted in a partial disappearance of the label and thus in a partial squeezing out of the SpA molecules from the film accompanied by a sudden rise but subsequent fall in the surface pressure. Reexpansion led to the initial surface pressure. However, if the subphase-isolated ELSSF was compressed, washed and re-expanded to the initial surface area, the surface pressure did not reach its initial value and almost all the gold label disappeared. On the contrary, films of lipid extracts of lung lavages or amniotic fluid spread over saline in the absence of proteins had unusually low values of the doublelayer capacitance $C\left(=1.1 \mu \mathrm{F} \mathrm{cm}^{-2}\right)$ and, after exceeding monolayer surface concentrations, no further rise in the surface pressure could be obtained. We must draw the most striking conclusion that the extracted lipids of that peculiar composition in which they were present in these biological products, after being spread at the air-saline interface, were not able to build a monolayer but a very stable multilayer-probably a trilayer. However, after any contact of the film with $\mathrm{SpA}$ or the lavage proteins, the surface pressure increased and $C$ rose from $1 \mu \mathrm{F} \mathrm{cm} \mathrm{cm}^{-2}$, attributed to a multilayer, to $C$ values of $3.4 \mu \mathrm{F} \mathrm{cm}^{-2}$, much higher than the $C$ values known for phospholipid monolayers $\left(1.7-1.9 \mu \mathrm{F} \mathrm{cm}^{-2}\right)$ (Fig. 2). The ELSSFs of the crude lavages had $C$ values twice as high $\left(6.2 \mu \mathrm{F} \mathrm{cm}^{-2}\right)$ as those

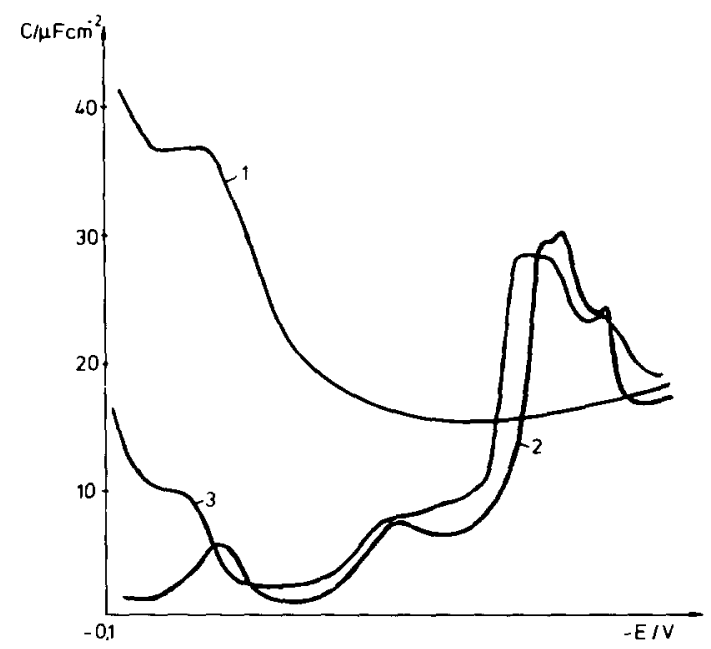

Fig. 2. Change in the electrical double-layer capacitance $C$ of lipid films in contact with surfactant proteins, as demonstrated by the $C-V$ curves of films at the air-water interface: curve 1, saline; curve 2 , amniotic-fluid-extracted lipids, surface concentration of $2 \mu \mathrm{g} \mathrm{cm}^{-2}$; curve 3, reassembled ELSSF after having injected surfactant-specific protein SpA $\left(1.25 \mu \mathrm{g} \mathrm{ml}^{-1}\right)$ beneath the lipid layer. 
of the ELSSFs of reassembled films. It should be mentioned that measurements carried out on films of crude lung lavages adsorbed in the bulk at the mercury-saline interface showed the same $C$ values as ELSSFs transferred from the air-saline to the HMDE-saline interface.The TEM images of the immuno-gold-labelled ELSSF specimens reassembled by shifting a lipid film over a protein solution in the trough showed that the proteins penetrated into the condensed lipid layer and could be cotransported with the lipids. We concluded therefore that a strong lipid-protein interaction favours SpA penetration into the condensed film of surfactant-building lipids, which results in a change of the lipid film molecularity; it switches from a multilayer to a loose monolayer.

Other evidence that lipid film-protein contact results in a more loose lipid layer was provided by the d.c. polarographic oxygen reduction measurements ${ }^{9}$. The halfpeak oxygen reduction potential found with a lipid layer was shifted in equilibrium with $200 \mathrm{mV}$ towards more positive values after SpA had been injected beneath the lipid film (Fig. 3). Thus the energetic barrier that the oxygen has to overcome to be reduced at the electrode was decreased by the protein integrated in the adsorbed film.

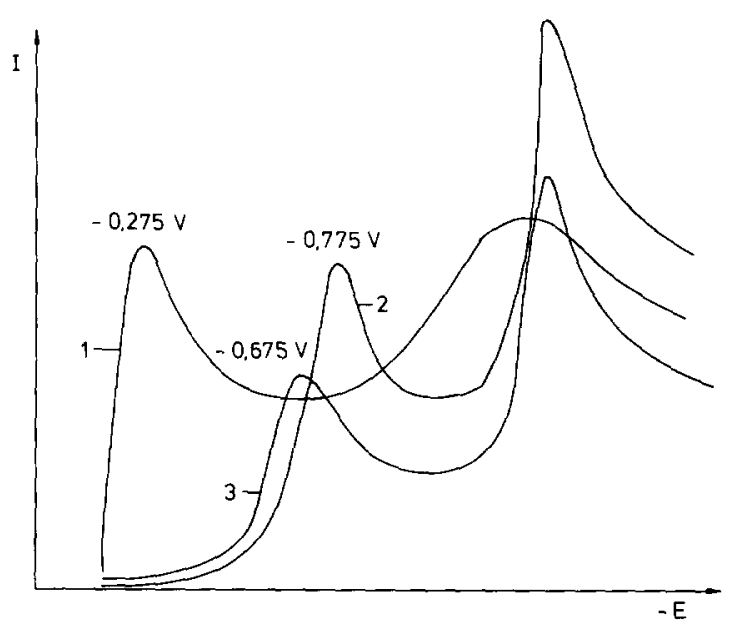

Fig. 3. Shift of the oxygen half-peak reduction potential depending on the film composition, as demonstrated by d.c.-potential curves of oxygen reduction at a hanging mercury drop electrocie without a lipid film (curve 1), with a lipid film (curve 2) and the SpA proteins injected beneath the lipid film (curve 3).

If all this is true, the question arises of what happened to the excess lipids when after contacting the proteins the multilayer switched to a loose monolayer? Doublelayer capacitance measurements carried out in the bulk showed that the capacitance of the film adsorbed in the bulk at the mercury-saline interface decreased when a preformed lipid film contacted proteins or when the lipids were spread over a protein solution. After $24 \mathrm{~h}$ the capacitance values at the surface and in the bulk fell at the same rate and became very close to each other (Fig. 4). This meant that some of the lipids transferred into the subphase. We interpret these results also in terms of the protein-lipid interaction and suggest that $\mathrm{SpA}$ is a lipid-transporting protein. 


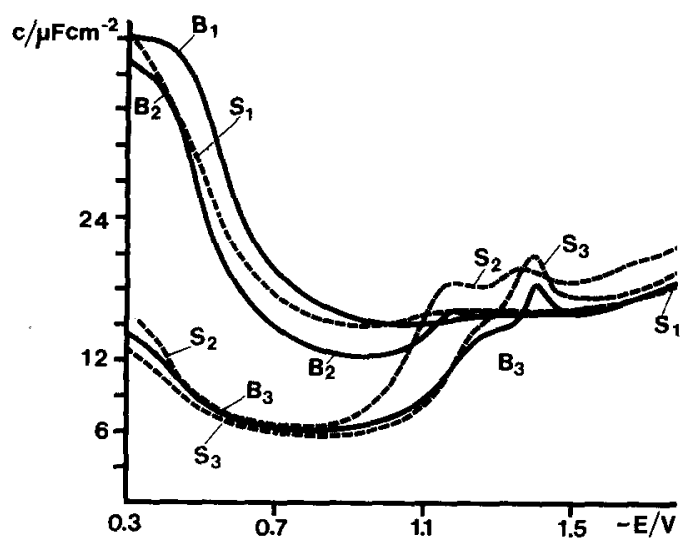

Fig. 4. Transport of surfactant lipid molecules spread at the air-saline interface into the bulk containing $1.25 \mu \mathrm{g} \mathrm{SpA}$ protein $\mathrm{ml}^{-1}$, as demonstrated by $C-V$ curves of the film in the bulk (B) and at the surface (S): curve 1, without lipids; curve 2, lipid extract spread onto the surface of the protein solution, $t=0 \mathrm{~h}$; curve 3 , lipid extract spread onto the surface of the protein solution, $t=7.5 \mathrm{~h}$.

These data indicate that the lipid-protein interaction can be important in the breathing process and allows us to propose a molecular mechanism explaining how the surfactant film works in the two phases of breathing. We suggest that compression of the film during expiration leads to a squeezing out of the SpA proteins which take with them the excess of lipid molecules into the subphase. This would assure the maintenance of a condensed lipid monolayer and a reasonable surface tension at a diminished alveolar area. During inspiration, SpA molecules penetrating the interface would bring with them the supplementary amount of lipid molecules from the subphase necessary for the full coverage of the augmented alveolar area.

Our results are consistent with data indicating that $\mathrm{SpA}$ interacts with surfactant lipids ${ }^{10}$, that it facilitates lipid adsorption ${ }^{10}$ and that it re-incorporates into surfactant-secreting cells ${ }^{11}$ and some lipid precursors ${ }^{12}$.

\section{CONCLUSION}

The ELSSF reassembled or isolated from lung lavages in vitro is a continuous film. The surfactant-specific protein molecules of type SpA are present in the ELSSF and are in a strong hydrophobic interaction mostly with its phospholipid components. The SpA presence confers to the ELSSF a loose monomolecular structure, and a proteinless film of the same lipid composition is a condensed multilayer. Owing to the strong hydrophobic interaction with the phospholipids, SpA molecules can play a lipid-carrier role. They can remove lipids in excess from the air-water interface into the aqueous subphase and vice versa. Since SpA molecules are squeezed out of the film when it is compressed, lipid-protein interaction can be of importance in vivo and can explain how the surfactant film works during the two phases of breathing. 


\section{ACKNOWLEDGMENT}

The first author acknowledges financial support of the Deutsche Forschungsgemeinschaft to carry out part of this research at the Weizmann Institute of Science, Rehovot, Israel.

\section{REFERENCES}

1 R. J. King, J.Appl. Physiol., 53 (1982) 1.

2 B. Benson, M. Williams and S. Hawgood, in P. von Wichert (ed.), Current Concepts in Surfactant Research, Karger, Basel, 1984, p. 83.

3 J. A. Clements, Proc. Soc. Exp. Biol. Med., 95 (1957) 170.

4 C. Jarstrand, in B. Robertson, L M. G. Van Golde and J. J. Batenburg (eds.), Pulmonary Surfactant, Elsevier, Amsterdam, 1984, p. 187.

5 E. Ladanyi, Prax. Klin. Pneumol., 40 (1986) 465.

6 E. Ladanyi, D. Möbius, K. Stalder and P. von Wichert, Actas Inst. Bioqim., to be published.

7 E. Ladanyi, I. Miller, R. Popovits-Biro, Y. Marikovsky, P. von Wichert, B. Müller and K. Stalder, Prog. Respir. Res., to be published.

8 M. Lecompte and I. R. Miller, Biochemistry, 19 (1980) 3439.

9 E. Ladanyi, Electrochemical behaviour of oxygen with lung surfactant, in Proc. Int. Symp. on OTT, Ottawa, 1988, Plenum, New York to be published.

10 R. King, M. Carmichael and P. Horowitz, J. Biol. Chem., 258 (1983) 10672.

11 J. Wright, S. Young, P. Stevens and J. Clements, Prog. Respir. Res, to be published.

12 J. Wright, R. Wager, R. Hamilton, M. Huang and J. Clements, J. Appl. Physiol., 60 (1986) 817. 